\title{
Análise da composição nutricional da Cesta Básica brasileira
}

\author{
Analysis of the nutritional composition \\ of the Brazilian Staple Foods Basket
}

Kelly Estarla dos Passos ${ }^{1}$

Juliana Rombaldi Bernardi ${ }^{1}$

Karina Giane Mendes ${ }^{1}$

${ }^{1}$ Centro de Ciências

Biológicas e da Saúde, Universidade de Caxias do Sul. R. Francisco Getúlio Vargas 1130, Petrópolis. 95.070-560 Caxias do Sul RS Brasil.

n.kellypassos@gmail.com
Abstract This study sought to assess the nutritional status (energy, carbohydrates, proteins, lipids, calcium, iron, zinc, phosphorus, vitamin A and vitamin $C$ ) of the 13 items in the Brazilian staple foods basket and to verify whether they are suitable for the monthly subsistence of an adult. The values established by the Estimated Average Requirement were compared to those found in the study. It is a descriptive study that used information from a food composition table in order to analyze data regarding the Brazilian staple foods basket from the Inter-Union Department of Statistics and Socio-Economic Studies. Appropriate values were found for carbohydrates, proteins, iron, zinc, phosphorus. Total energy and lipids were above the recommended values, while calcium and vitamin A were below. Vitamin $C$ had adequate values only for females. The Brazilian staple foods basket largely reflects the individual food intake. However, given the prevalence of obesity in the Brazilian population, some adjustments in this nutritional basis are required, so that it is rendered adequate in terms of macronutrient and micronutrient values.

Key words Nutritional value, Food products, Nutritional requirements, Food consumption, Food analysis, Staple Foods
Resumo O presente estudo teve como objetivo analisar o valor nutricional (energia, carboidratos, proteínas, lipídeos, cálcio, ferro, zinco, fósforo, vitamina A e vitamina $C$ ) dos treze itens alimentares que compõem a Cesta Básica Brasileira e verificar se estão adequados para a subsistência mensal de um indivíduo adulto. Foi realizada a comparação dos valores encontrados com os estabelecidos pela Estimated Average Requirement. Trata-se de um estudo descritivo que utilizou informações de uma tabela de composição centesimal de alimentos para a análise dos dados relativos da Cesta Básica Brasileira proveniente do Departamento Intersindical de Estatística e Estudos Socioeconômicos. Foram encontrados valores adequados para carboidratos, proteínas, ferro, zinco, fósforo. Valor energético total e lipídeos estavam acima do recomendado, enquanto Cálcio e Vitamina A estavam abaixo. A Vitamina $C$ teve valores adequados apenas para o sexo feminino. Foi possível observar que a Cesta Básica reflete em grande parte o consumo alimentar dos indivíduos, porém, diante da prevalência de obesidade da população brasileira, seriam necessárias algumas modificações nesta base alimentar para que esta fique adequada em macronutrientes e micronutrientes.

Palavras-chave Valor nutricional, Alimentos, Necessidades Nutricionais, Consumo alimentar, Análise de alimentos, Alimentação básica 


\section{Introdução}

Os estudos referentes à alimentação da população brasileira iniciaram a partir da primeira metade do século XIX. Os pioneiros nesses estudos questionavam em geral a relação entre a pobreza e a alimentação da população. Os primeiros autores a realizar e publicar estudos referentes a este tema foram: Francisco F. Padilha em 1842, Antônio J. Sousa em 1851, Antônio C. Costa em 1851, Josué Castro em 1932, e novamente em 1934, Ruy Coutinho em 1937 e Dante N. Costa em $1938^{1 .}$

No ano de 1938 foi sancionado o Decreto Lei n 399 que regularizava o salário mínimo e, em conjunto, estabelecia o direito a chamada Ração Essencial Mínima (Cesta básica nacional), na qual foram listados treze itens alimentares e suas quantidades, considerados básicos para a subsistência nutricional de um trabalhador e sua família (total de quatro pessoas) ${ }^{2}$.

Essa cesta básica nacional, composta de 13 itens, foi formulada a partir dos dados obtidos em estudos da década de $1930^{1}$ e tinha como objetivo ser suficiente para o sustento e bem estar de um trabalhador em idade adulta, contendo quantidades balanceadas de calorias, proteínas, ferro cálcio e fósforo ${ }^{2}$. A Ração Essencial Mínima originou outras cestas básicas, municipais, estaduais e regionais, cada uma levando em consideração o poder econômico e a cultura da população local. Porém, observa-se o fato de que, até hoje, os alimentos contidos na Ração Essencial Mínima, servirem como base da alimentação brasileira ${ }^{3}$.

Atualmente o Departamento Intersindical de Estatística e Estudos Sócioeconômicos (DIEESE) realiza mensalmente em 16 capitais brasileiras uma pesquisa referente ao custo que os 13 itens alimentares da Cesta Básica Nacional tem para os trabalhadores, e compara os dados com o valor do salário mínimo vigente. É importante salientar que somente o custo da Cesta Básica Nacional é calculado, não existindo dados referentes ao seu valor nutricional, quanto à macronutrientes e micronutrientes ${ }^{4}$.

No Brasil, existem realidades populacionais bem distintas. Enquanto algumas famílias ficam restritas aos itens da Cesta Básica, outras acabam tendo opções alimentares adicionais no consumo diário. Porém, independentemente da condição social e cultural, os itens que compõe a Ração Essencial Mínima, permanecem como sendo a base alimentar da população brasileira ${ }^{3,5,6}$. A cesta básica nacional é composta por treze itens alimentares: carne, leite, feijão, arroz, farinha, batata, legumes (tomate), pão francês, café em pó, frutas (banana), açúcar, óleo e manteiga ${ }^{4}$.

Dados da Pesquisa de Orçamentos Familiares 2008-2009 (POF) ${ }^{6}$ demonstrou que os itens da Cesta Básica Nacional têm grande relevância na alimentação rotineira da população brasileira. A partir dessa pesquisa, é possível obter os dados referentes à quantidade (em quilogramas) de alimentos adquiridos por pessoa durante o período de um ano. Com esses dados, pode-se perceber que os alimentos da Cesta Básica são os adquiridos em maior quantidade dentro de seus respectivos grupos, sendo consequentemente os mais consumidos pela população ainda na atualidade ${ }^{6}$.

Considerando que não está claro na literatura se a Cesta Básica Nacional é capaz de suprir as necessidades nutricionais de um indivíduo adulto saudável comparado às recomendações de ingestão dietética, este trabalho teve como objetivo analisar o valor nutricional (energia, carboidratos, proteínas, lipídeos, cálcio, ferro, zinco, fósforo, vitamina A e vitamina C) dos treze itens alimentares que compõem a cesta básica nacional e verificar se esses estão adequados para a subsistência mensal de um indivíduo adulto.

\section{Metodologia}

Trata-se de um estudo transversal descritivo que utilizou informações de uma tabela de composição centesimal de alimentos para a análise dos dados relativos da Cesta Básica Nacional proveniente do DIEESE, através do seu endereço eletrônico ${ }^{4}$.

A Tabela 1 mostra os resultados referentes ao cálculo dos itens da Cesta Básica Brasileira, divididos por trinta dias, para refletir o consumo diário para um indivíduo adulto. Através do DIEESE é possível obter os dados referentes às quantidades per capitas mensais de cada item da cesta. A partir desses dados, foi possível determinar as quantidades de per capitas diários, estimados para cada alimento: carne, $200 \mathrm{~g}$; leite, $500 \mathrm{ml}$; feijão, 150g; arroz, 100g; farinha de trigo, 50g; batata, 200g; legumes (tomate), 300g; pão francês, 200g; café em pó, 20g; frutas (banana), 3 unidades; açúcar, 100g; óleo, 50ml; manteiga, 30g.

Os dados para os cálculos foram retirados da Tabela de Composição Centesimal de Alimentos do Instituto Brasileiro de Geografia e Estatística (IBGE) de $2011^{7}$. O item alimentar "farinha de trigo" foi calculado a partir dos dados obtidos na Tabela Brasileira de Composição de Alimen- 
Tabela 1. Quantidade de alimentos per capita para consumo diário da população brasileira conforme cesta básica.

\begin{tabular}{lc}
\hline \multicolumn{1}{c}{ Alimento } & Quantidade $(\mathrm{g} \mathrm{ou} \mathrm{ml})$ \\
\hline Carne & $200 \mathrm{~g}$ \\
Leite Integral & $500 \mathrm{ml}$ \\
Feijão & $150 \mathrm{~g}$ \\
Arroz & $100 \mathrm{~g}$ \\
Farinha de trigo & $50 \mathrm{~g}$ \\
Batata & $200 \mathrm{~g}$ \\
Legumes (tomate) & $300 \mathrm{~g}$ \\
Pão francês & $200 \mathrm{~g}$ \\
Café em pó & $20 \mathrm{~g}$ \\
Frutas (banana) & $210 \mathrm{~g}$ \\
Açúcar & $100 \mathrm{~g}$ \\
Óleo & $50 \mathrm{ml}$ \\
Manteiga & $30 \mathrm{~g}$ \\
\hline
\end{tabular}

$\mathrm{ml}$ - mililitros; g - gramas.

tos (TACO) de $2011^{8}$, por falta de dados na Tabela do IBGE.

Os itens: feijão, arroz, batata, tomate e banana, foram calculados conforme o valor médio descrito na tabela de composição de alimentos, devido à diversidade que foi encontrada dos mesmos na tabela. O valor nutricional do óleo foi calculado através do óleo de soja presente na tabela centesimal de composição de alimentos. Para o item carne foi utilizado o valor médio descrito na tabela para carne bovina. No cálculo da banana, por ser divulgada em valor unitário pelo DIEESE, foi utilizada a gramatura média de cada unidade, conforme o descrito na Tabela para Avaliação de Consumo Alimentar em Medidas Caseiras $^{9}$. Para o cálculo do valor nutricional do leite foi utilizada a versão integral, por ser a mais consumida pela população brasileira segundo dados da POF (2008-2009) ${ }^{6}$.

Após a coleta dos dados referentes à cesta básica, foram realizados os cálculos do valor nutricional dos alimentos que a compõe. Foram calculados os valores de energia total, carboidratos, proteínas, lipídeos, ferro, cálcio, zinco, fósforo, vitamina A e vitamina C. Os valores de macronutrientes estão descritos em percentuais, já os valores de micronutrientes estão descritos conforme sua respectiva unidade de medida: ferro, zinco, cálcio, fósforo e vitamina C em miligramas (mg) e a vitamina A em microgramas $(\mu \mathrm{g})$.

$\mathrm{O}$ valor energético total foi calculado a partir dos resultados obtidos de carboidratos, proteínas e lipídeos. Os valores de carboidratos e proteínas (em gramas) foram multiplicados por 4 kcal por grama e o valor de lipídeos foi multiplicado por $9 \mathrm{kcal}$ por grama. Após, os três valores encontrados foram somados, resultando assim no valor calórico total da Cesta Básica Brasileira. O valor calórico total foi comparado com o recomendado pelo Guia Alimentar para a População Brasileira ${ }^{10}$.

A quantidade de nutrientes encontrados foi comparada com as recomendações de ingestão dietética de 2010 (DRI, do inglês Dietary Reference Intake $)^{11}$ por meio dos valores das estimativas do requerimento médio (EAR, do inglês Estimated Average Requerement), para um adulto saudável.

\section{Resultados}

A Tabela 2 mostra os resultados referentes aos valores de carboidratos, proteínas, lipídeos, ferro, zinco, cálcio, fósforo, vitamina A e vitamina C, para um dia de consumo de um indivíduo adulto a partir dos dados da Cesta Básica e a comparação com os valores preconizados pela EAR (DRI, 2010) ${ }^{11}$.

A análise de macronutrientes resultou em valores adequados, porém próximos ao limite mínimo, para o consumo segundo a EAR, para carboidratos e proteínas. A Cesta Básica está composta de 45,14\% de carboidratos, (EAR 45\% a $65 \%$ ) e $10,43 \%$ de proteínas (EAR 10\% a 35\%). No cálculo de lipídeos foi encontrado o valor de $44,42 \%$, estando quase $10 \%$ acima dos percentis recomendados pela EAR $(20 \%$ a $35 \%)$. Nos cálculos de micronutrientes, foram obtidos valores adequados para o consumo de ambos os sexos de ferro, zinco e fósforo.

O valor de vitamina $\mathrm{C}$ encontrado na Cesta Básica foi de 71,17 mg, atingindo os valores estabelecidos apenas para indivíduos do sexo feminino. A EAR para este nutriente é de $60 \mathrm{mg}$ por dia para o sexo feminino e $75 \mathrm{mg}$ por dia para o sexo masculino. Para os indivíduos do sexo masculino, o valor encontrado está $5,11 \%$ abaixo do mínimo recomendado. O cálcio tem como quantidade mínima de $800 \mathrm{mg}$ para o sexo masculino (19 aos 70 anos) e também para o sexo feminino (19 aos 51 anos) e $1000 \mathrm{mg}$ para o sexo feminino na faixa etária dos 51 aos 70 anos. As análises deste nutriente resultaram em 727,66 mg de cálcio por dia, estando 9,04\% abaixo da recomendação mínima dos $800 \mathrm{mg}$ e 27,23\% abaixo das necessidades de $1000 \mathrm{mg}$ para o sexo feminino de 51 aos 70 anos. Foram encontrados 479,371 $\mu \mathrm{g}$ de vitamina A, estando 23,30\% abaixo das necessidades diárias $(625 \mu \mathrm{g})$ para indivíduos do sexo 
Tabela 2. Valores de nutrientes encontrados na análise da Cesta Básica Nacional.

\begin{tabular}{|c|c|c|c|c|c|c|}
\hline \multirow{2}{*}{ Nutrientes } & \multicolumn{2}{|c|}{$\begin{array}{l}\text { Sexo masculino } \\
\text { (19 a } 70 \text { anos })\end{array}$} & \multicolumn{2}{|c|}{$\begin{array}{l}\text { Sexo feminino } \\
(19 \text { a } 50 \text { anos })\end{array}$} & \multicolumn{2}{|c|}{$\begin{array}{l}\text { Sexo feminino } \\
(51 \text { a } 70 \text { anos })\end{array}$} \\
\hline & EAR & Cesta & EAR & Cesta & EAR & Cesta \\
\hline Carboidrato & $45 \%$ a $65 \%$ & $45,14 \%$ & $45 \%$ a $65 \%$ & $45,14 \%$ & $45 \%$ a $65 \%$ & $45,14 \%$ \\
\hline Proteína & $10 \%$ a $35 \%$ & $10,43 \%$ & $10 \%$ a $35 \%$ & $10,43 \%$ & $10 \%$ a $35 \%$ & $10,43 \%$ \\
\hline Lipídio & $20 \%$ a $35 \%$ & $44,42 \%$ & $20 \%$ a $35 \%$ & $44,42 \%$ & $20 \%$ a $35 \%$ & $44,42 \%$ \\
\hline Ferro & $6 \mathrm{mg}$ & $\begin{array}{c}13,699 \mathrm{mg} \\
(\uparrow 128,316 \%)\end{array}$ & $8,1 \mathrm{mg}$ & $\begin{array}{l}13,699 \mathrm{mg} \\
(\uparrow 69,12 \%)\end{array}$ & $5 \mathrm{mg}$ & $\begin{array}{c}13,699 \mathrm{mg} \\
(\uparrow 173,98 \%)\end{array}$ \\
\hline Zinco & $9,4 \mathrm{mg}$ & $\begin{array}{l}18,556 \mathrm{mg} \\
(\uparrow 97,40 \%)\end{array}$ & $6,8 \mathrm{mg}$ & $\begin{array}{c}18,556 \mathrm{mg} \\
(\uparrow 172,88 \%)\end{array}$ & $6,8 \mathrm{mg}$ & $\begin{array}{c}18,556 \\
(\uparrow 172,88 \%)\end{array}$ \\
\hline Cálcio & $800 \mathrm{mg}$ & $\begin{array}{c}727,66 \mathrm{mg} \\
(\uparrow 9,04 \%)\end{array}$ & $800 \mathrm{mg}$ & $\begin{array}{c}727,66 \mathrm{mg} \\
(\uparrow 9,04 \%)\end{array}$ & $1000 \mathrm{mg}$ & $\begin{array}{l}727,66 \mathrm{mg} \\
(\uparrow 27,23 \%)\end{array}$ \\
\hline Fósforo & $580 \mathrm{mg}$ & $\begin{array}{l}1420,17 \mathrm{mg} \\
(\uparrow 144,86 \%)\end{array}$ & $580 \mathrm{mg}$ & $\begin{array}{l}1420,17 \mathrm{mg} \\
(\uparrow 144,86 \%)\end{array}$ & $580 \mathrm{mg}$ & $\begin{array}{l}1420,17 \mathrm{mg} \\
(\uparrow 144,86 \%)\end{array}$ \\
\hline Vitamina A & $625 \mu \mathrm{g}$ & $\begin{array}{l}479,371 \mu g \\
(\uparrow 23,30 \%)\end{array}$ & $500 \mu \mathrm{g}$ & $\begin{array}{c}479,371 \mu \mathrm{g} \\
(\uparrow 4,12 \%)\end{array}$ & $500 \mu \mathrm{g}$ & $\begin{array}{c}479,371 \mu \mathrm{g} \\
(\uparrow 4,12 \%)\end{array}$ \\
\hline Vitamina $\mathrm{C}$ & $75 \mathrm{mg}$ & $\begin{array}{l}71,17 \mathrm{mg} \\
(\uparrow 5,11 \%)\end{array}$ & $60 \mathrm{mg}$ & $\begin{array}{l}71,17 \mathrm{mg} \\
(\uparrow 18,62 \%)\end{array}$ & $60 \mathrm{mg}$ & $\begin{array}{l}71,17 \mathrm{mg} \\
(\uparrow 18,62 \%)\end{array}$ \\
\hline
\end{tabular}

mg-miligramas; $\mu \mathrm{g}-$ microgramas.

masculino e $4,12 \%$ abaixo dos valores mínimos estimados $(500 \mu \mathrm{g})$ para o sexo feminino.

$\mathrm{O}$ valor calórico total fornecido pela Cesta Básica Nacional, segundo os cálculos foi de $3731,35 \mathrm{kcal}$ por dia. A recomendação do Guia Alimentar para a População Brasileira é de que a ingestão calórica média para indivíduos adultos seja de $2000 \mathrm{kcal}$ por dia. A Cesta Básica estaria fornecendo $89,06 \%$ a mais de valor energético do que a média recomendada.

\section{Discussão}

No ano de 1938, quando foi aprovado o Decreto Lei $n^{\circ} 399^{2}$, que regulamentava o direito ao Salário Mínimo para os trabalhadores Brasileiros, foi também estabelecido que este deveria ser suficiente para a aquisição de uma lista de alimentos, designada de Ração Essencial Mínima, considerados como suficientes para a subsistência de uma família de quatro pessoas (dois adultos e duas crianças) durante o período de um mês ${ }^{4}$. Desde então, essa lista de alimentos continua sendo utilizada como modelo para pesquisas referentes ao custo que os itens alimentares têm no orçamento mensal da população. Essas pesquisas referentes ao custo da Cesta Básica acabam por influenciar a população nas escolhas que realizam ao adquirir os itens para a sua alimentação diária ${ }^{3}$.
Apesar dos alimentos que compõem a Cesta Básica permanecerem como os mais consumidos pela população brasileira desde a sua legalização, observa-se o fato de o perfil nutricional e de trabalho da população ter mudado intensamente $^{6}$. Até a metade do século XX, a população brasileira exercia predominantemente atividades profissionais rurais, braçais e com pouquíssimo maquinário que automatizasse as atividades realizadas, exigindo assim uma alimentação com valor energético relativamente alto ${ }^{12,13}$. Até 1950 , o índice de residentes na zona rural era de $66 \%$. A partir da década de 1950, iniciou uma notável migração do ambiente rural para o urbano, sendo que no Censo de 2010 foi constatado que $84 \%$ da população brasileira reside em território urbano $^{14}$. Nessa mesma época, iniciou-se também uma reestruturação produtiva, na qual cresceu a substituição do trabalho humano braçal por tecnologias automatizadas, que repercutia, entre outras questões, na diminuição das atividades de alto gasto calórico realizadas diariamente pelos trabalhadores brasileiros ${ }^{15}$.

Neste mesmo período (de alterações no perfil de trabalho e estilo de vida) houve mudanças no perfil nutricional da população brasileira em geral. Na época da implantação da Cesta Básica, alguns pesquisadores falavam sobre os problemas nutricionais no País, como desnutrição e carências de micronutrientes. Atualmente, ainda são encontrados problemas de desnutrição no 
país, porém esse já não é mais o quadro geral da população brasileira. O perfil nutricional da população mudou de desnutrição para sobrepeso e obesidade e houve um aumento das doenças crônicas não transmissíveis. É possível relacionar essa transição ao fato de ter diminuído o tempo em que os indivíduos passam realizando atividades que exijam esforço físico (mudanças no perfil profissional e estilo de vida) e ao mesmo tempo continuar com a mesma base alimentar da época em que a lei da Cesta Básica foi implantada, além do aumento do consumo de alimentos industrializados baratos e com alta densidade energética ${ }^{1,16}$.

O Guia Alimentar para a População Brasilei$\mathrm{ra}^{10}$ tem como indicação o valor médio de ingestão calórica para adultos de ambos os sexos de $2000 \mathrm{kcal}$ por dia. O valor calórico diário da Cesta Básica para um indivíduo adulto ficou $89 \%$ acima das necessidades médias diárias. Bonomo et al. ${ }^{17}$, em estudo sobre consumo alimentar de uma população adulta da cidade de Bambuí no Estado de Minas Gerais (MG), encontraram diferenças significativas entre os valores de energia total consumida entre homens e mulheres independentemente da renda familiar. A média de valor energético diário foi de 2807,4 kcal ( \pm $1537,80)$ para mulheres e $3774,9 \mathrm{kcal}( \pm 2183,9)$ para homens. Em comparação com os resultados da Cesta Básica, pode-se perceber que as mulheres da pesquisa ingeriram em média valores calóricos menores e os homens relataram consumo energético diário total maior, mas ambos ficaram acima do recomendado pelo Guia Alimentar para a População Brasileira ${ }^{10}$.

Os valores de carboidratos e proteínas da Cesta Básica estão dentro dos valores preconizados, porém, nota-se que estes estão próximos ao limite mínimo necessário por dia de consumo, sendo questionável se a população realmente consome as quantidades indicadas de macronutrientes pelas DRIS. Já os lipídeos estão bem acima do valor máximo preconizado para um indivíduo adulto. Quanto ao alto índice de lipídeos, este foi proveniente principalmente da alta quantidade de óleo previsto por dia de consumo (50 ml). É possível que a população não utilize essa quantidade de óleo em um único dia de consumo, sendo esta uma média do valor mensal total que é de $1500 \mathrm{ml}$ por mês, e a quantidade pode estar prevista para a utilização nas preparações em geral e também para as frituras, sendo que nestas, apesar de o alimento absorver a gordura, não é realizado o consumo de todo o óleo utilizado. É importante levar em consideração que, em geral, a população não fica restrita ao consumo dos itens da Cesta Básica, complementando a alimentação com outros itens. Muitas vezes essas escolhas são feitas por alimentos industrializados, de baixo custo, mas com alto valor calórico e de gordura, sódio e baixa quantidade de vitaminas e sais minerais. Na literatura atual, existem muitas evidências de que o alto consumo de açúcar, gorduras, farináceos e sódio são fatores de risco para o desenvolvimento de doenças crônicas não transmissíveis, como por exemplo, obesidade, diabetes melitus tipo 2, hipertensão arterial sistêmica, entre outras ${ }^{18}$. Os estudos também evidenciam que quanto mais baixa a condição socioeconômica da população, maior é o índices de consumo dos alimentos de alto valor energético e baixo valor nutricional para micronutrientes ${ }^{19,20}$.

Estudo na cidade de Bambuí, realizado por Lopes et al. ${ }^{21}$, pesquisou o consumo alimentar em uma população de 550 indivíduos para avaliar a ingestão de macro e micronutrientes. A ingestão de proteínas ficou em 59,5\% da população acima do recomendado. Os carboidratos foram descritos como $97,5 \%$ da população ingerindo-os abaixo dos valores preconizados. Já os lipídeos tiveram como resultado de 68,9\% da população com a ingestão diária adequada deste nutriente, sendo que não houve ingestão abaixo do recomendado.

É possível perceber um perfil de alimentação baseado quase que exclusivamente em itens da Cesta Básica no estudo realizado por Ferreira e Magalhães ${ }^{22}$, com mulheres de baixa renda, moradoras da Favela da Rocinha (Rio de Janeiro), onde foi relatado o alto consumo dos itens como arroz, feijão, açúcar, farinhas e gorduras. Os itens como legumes foram ditos como raramente consumidos, exceto os tubérculos como a batata, que era consumida frequentemente. Outro fato interessante demonstrado neste estudo é as mulheres entrevistadas serem todas obesas, o que pode ser explicado pelo alto consumo de alimentos ricos em carboidratos e gorduras e pouca atividade física, fazendo com que tenham ingestão diária de alta densidade calórica. Neste estudo, foi muito presente a questão da alimentação para "encher a barriga" (relato das participantes) e que as frutas, verduras e legumes não teriam tanta importância, por proporcionarem menor saciedade. No que diz respeito à carne, a Cesta Básica tem como quantidade 200 g diárias por pessoa. No estudo da Favela da Rocinha as moradoras relataram consumir a carne praticamente todos os dias, porém, somente a carne de fran- 
go, por ter o custo menor. A carne de gado era utilizada raramente. Porém, é questionável se pessoas carentes, que tenham acesso praticamente só a carne de frango, consigam garantir os valores mínimos necessários para a prevenção de patologias provenientes da deficiência de ferro.

$\mathrm{Na}$ Cesta Básica pode-se observar que as quantidades de ferro estão adequadas conforme as necessidades diárias de um adulto. As maiores quantidades de ferro foram provenientes da carne, seguida do feijão e do pão francês. Na literatura pesquisada, foram encontrados valores adequados de ferro, porém nem sempre são descritas as fontes alimentares desse nutriente (animal ou vegetal). É importante ressaltar que nem toda a população tem por hábito o consumo da carne vermelha diariamente, sendo feita a substituição muitas vezes, pela carne de frango (menor teor de ferro). A quantidade de carne da Cesta Básica (200 g por dia) pode estar sendo superestimada, pois os indivíduos mais restritos aos itens da Cesta são na maioria de baixa renda, e nessa classe econômica, o consumo da carne acaba sendo menor, devido ao custo da mesma ${ }^{23}$. Também foram verificadas quantidades relevantes de ferro no feijão e no pão francês, porém no feijão é encontrado ferro não heme (origem vegetal) e o ferro do pão francês é o adicionado na farinha de trigo.

Pesquisa realizada em serviço de saúde do Estado de São Paulo, com mulheres em idade reprodutiva, encontrou que o consumo de ferro das entrevistadas estava adequado, porém, esse ferro era em sua maioria proveniente de fontes vegetais e alimentos fortificados (farinha de trigo), sendo que o ferro de origem animal era consumido em menores proporções que o de origem vegetal. Neste mesmo estudo, foram encontrados valores adequados de consumo de Vitamina C. Nos cálculos da Cesta Básica, foram encontrados valores adequados para o sexo feminino e inadequados para o sexo masculino de vitamina C. É importante enfatizar que para a absorção do ferro pelo organismo humano ocorrer de forma adequada, são necessários níveis adequados de consumo de Vitamina $\mathrm{C}^{24}$.

Nos resultados referentes à Vitamina A e Cálcio, pode-se notar que a composição da Cesta não consegue suprir as necessidades diárias de um adulto para estes micronutrientes. As carências de vitamina A estão entre os problemas nutricionais mais resistentes no país, principalmente nas populações de baixa renda ${ }^{25}$. Na pesquisa realizada por Lopes et al. ${ }^{21}$, foi constatado que 99,8\% indivíduos entrevistados tiveram consu- mo de Vitamina A abaixo dos valores mínimos necessários. A deficiência de cálcio mostra-se presente na população adulta tanto em países desenvolvidos como nos em desenvolvimento, independentemente do nível socioeconômico. Esta deficiência é mais comum entre as mulheres, e é justamente para o sexo feminino que esta situação torna-se mais preocupante, devido ao risco aumentado para o desenvolvimento de osteoporose. Muitos estudos mostram o consumo inadequado de cálcio em adultos, principalmente no sexo feminino ${ }^{24-26}$. Montilla et al. ${ }^{27}$ em pesquisa realizada com mulheres no período do climatério observou a ingestão insuficiente de cálcio em mais de $85 \%$ da população estudada $(n=154)$. Lopes et al. ${ }^{21}$ encontrou valores ainda maiores na inadequação do consumo de cálcio em $98 \%$ da população estudada ( $n=660$ de ambos os sexos). Estudo realizado na Polônia, por Ilow et al. ${ }^{28} \mathrm{com}$ 3862 participantes de ambos os sexos, avaliou a ingestão de vitaminas e minerais e encontrou valores de ingestão de cálcio abaixo do recomendado em $90 \%$ da população pesquisada.

Nos cálculos para os nutrientes zinco e fósforo, foram encontrados valores satisfatórios conforme o preconizado pela EAR. Em suas diretrizes, o DIEESE ${ }^{4}$ estabelece que a Cesta Básica deve possuir quantidades balanceadas de fósforo. Ambos foram provenientes principalmente da alta quantidade de carne prevista na composição da Cesta. Estudo de Suliburska ${ }^{26}$ avaliou a ingestão diária de minerais e as concentrações de minerais no cabelo em setenta e sete mulheres adultas. Em ambos os testes foram encontrados valores adequados de zinco, não demonstrando deficiências deste nutriente na população estudada.

Pode-se questionar se os micronutrientes que não foram calculados neste estudo estariam sendo supridos através da cesta básica ou se esta estaria ofertando valores abaixo dos recomendados para os indivíduos adultos.

Como limitação deste estudo é possível citar o fato de que os itens da cesta básica são descritos em sua forma crua e existem diversas formas de preparo e utilização de cada um deles, estando presentes as questões culturais de cada região do país e a possibilidade de haver desperdício de alimentos. Também nota-se que a cesta básica, em suas quantidades originais previstas na Lei $\mathrm{N}^{\circ}$ 399 de $1938^{2}$, tem valores padronizados para uma família de quatro pessoas e cada um dos integrantes teria direito a partes iguais no consumo dos alimentos, porém não se sabe ao certo as quantidades de alimentos que cada indivíduo consome em seu respectivo núcleo familiar. 
Com base na literatura consultada e nos resultados obtidos nos cálculos nutricionais da Cesta Básica Nacional, pode-se dizer que esta reflete em grande parte o consumo alimentar dos indivíduos. Porém, diante do quadro nutricional atual da população brasileira, seriam necessárias algumas modificações nesta base alimentar, como por exemplo, a diminuição da quantidade total de gorduras e o aumento de alimentos fontes de cálcio e vitamina A. Para combater o excesso de peso na população, nota-se a importância da redução dos preços de itens alimentares que não fazem parte da Cesta Básica, mas que a complementariam, melhorando seu valor nutricional (frutas, verduras, legumes), diminuindo o valor calórico e aumentando as quantidades de vitaminas e sais minerais. $\mathrm{O}$ alto consumo de óleo na alimentação do brasileiro pode ser uma das causas da obesidade. Se o mesmo fosse reduzido das preparações, e com o valor fossem adquiridos outros alimentos (frutas verduras, legumes), a dieta se tornaria mais saudável, suprindo assim as necessidades nutricionais reais dos indivíduos. Ainda hoje os indivíduos de baixa condição socioeconômica continuam sendo os mais atingidos pelas carências de micronutrientes. Destaca-se também a importância de estratégias de educação nutricional para a população, focando em melhores escolhas alimentares, tanto em qualidade como em quantidade.

\section{Colaboradores}

KE Passos participou da concepção, coleta, análise e interpretação dos dados; JR Bernardi trabalhou na metodologia, fez a revisão crítica e aprovou a versão a ser publicada; KG Mendes auxiliou na análise e interpretação dos dados e aprovou a versão a ser publicada. 


\section{Referências}

1. Vasconcelos FAG. Combate à fome no Brasil: uma análise histórica de Vargas a Lula. Rev Nutr 2007; 18(4):439-457.

2. Brasil. Decreto Lei $\mathrm{n}^{\circ} 399$, de 30 de outubro de 1938. Diário Oficial da República Federativa do Brasil. Poder Executivo, Brasília, DF, 30 out. 1938.

3. Barretto SAJ, Cyrillo DC, Cozzolino SMF. Análise nutricional e complementação alimentar de cesta básica derivada do consumo. Rev Saude Publica 1998; 32(1):29-35.

4. Departamento Intersindical de Estatística e Estudos Socioeconômicos (DIEESE). Cesta básica nacional: metodologia. 1993. [Internet]. [acessado 2013 mar 18]. Disponível em: http://www.dieese.org.br/ $\mathrm{rel} / \mathrm{rac} / \mathrm{metodologia.pdf}$

5. Nascimento S, Barbosa FS, Sichieri R, Pereira, RA. Dietary availability patterns of the brazilian macroregions. Nutrition J 2011; 10:79.

6. Instituto Brasileiro de Geografia e Estatística (IBGE). Pesquisa de orçamentos Familiares. Aquisição alimentar domiciliar per capita Brasil e Grandes Regiões: Período 2008 - 2009. Rio de Janeiro, 2010. [Internet]. [acessado 2013 mar 18]. Disponível em: http:/ /www.ibge.gov.br/home/estatistica/populacao/ condicaodevida/pof/2008_2009_aquisicao/pof2008 2009 aquisicao.pdf

7. Instituto Brasileiro de Geografia e Estatística (IBGE). Pesquisa de orçamentos Familiares. Tabela de Composição Nutricional dos Alimentos Consumidos no Brasil. Rio de Janeiro: IBGE; 2011. [Internet]. [acessado 2013 mar 10]. Disponível em: http://www. ibge.gov.br/home/estatistica/populacao/condicaodevida/pof/2008_2009_composicao_nutricional/ pofcomposicao.pdf

8. Universidade Estadual de Campinas (Unicamp). Tabela Brasileira de Composição de Alimentos (TACO). $4^{\mathrm{a}}$ ed. Campinas: Unicamp; 2011.

9. Pinheiro ABV, Lacerda, EMA, Benzecry EH, Gomes MCS, Costa VM. Tabela para avaliação de consumo alimentar em medidas caseiras. $5^{\mathrm{a}}$ ed. São Paulo: Atheneu; 2005.

10. Brasil. Ministério Da Saúde (MS). Guia Alimentar para a população brasileira: promovendo a alimentação saudável. Brasília: MS; 2005. (Série A. Normas e manuais técnicos).

11. Institute of Medicine (US). Committee to Review Dietary Reference Intakes for Vitamin D and Calcium. In: Ross AC, Taylor CL, Yaktine AL, Del Valle $\mathrm{HB}$, editors. Dietary Reference Intakes for calcium and vitamin D. Washington: National Academies Press (US); 2010.

12. Patarra LP. Mudanças na dinâmica demográfica. In: Monteiro CA, organizador. Velhos e Novos $\mathrm{Ma}$ les da Saúde no Brasil. 2a ed. São Paulo: Editora Hucitec; 2000. p. 61-78

13. Batista Filho M, Rissin A. A transição nutricional no Brasil: tendências regionais e temporais. Cad Saude Publica 2003; 19(Supl. 1):S181-S191.

14. Instituto Brasileiro de Geografia e Estatística (IBGE). Censo 2010. Rio de Janeiro: IBGE. [Internet]. [acessado 2013 abr 02]. Disponível em: http://censo2010. ibge.gov.br/resultados
15. Wunsch Filho V. Perfil Epidemiológico dos Trabalhadores. Rev Bras Med Trab 2004; 2(2):103-117.

16. Pinheiro ARO, Freitas SFT, Corso ACT. Uma abordagem epidemiológica da obesidade. Rev Nutr 2004; 17(4):523-533.

17. Bonomo E, Caiaffa WT, César CC, Lopes ACS, Limacosta M F. Consumo alimentar da população adulta segundo perfil sócio-econômico e demográfico: projeto bambuí. Cad Saude Publica 2003; 19(5):1461-1471.

18. Neumann AICP, Martins IS, Marcopito LF, Araujo EAC. Padrões alimentares associados a fatores de risco para doenças cardiovasculares entre residentes de um município brasileiro. Rev Panam Salud Publica 2007; 22(5):329-339.

19. Sarti FM, Claro RM, Bandoni DHB. Contribuições de estudos sobre demanda de alimentos à formulação de políticas públicas de nutrição. Cad Saude Publica 2011; 27(4):639-647.

20. Lenz A, Olinto MTA, Dias-da-Costa JS, Alves AL, Balbinotti M, Patussi MP, Bassani DG. Socioeconomic, demographic and lifestyle factors associated with dietary patterns of women living in Southern Brazil. Cad Saude Publica 2009; 25(6):1297-1306.

21. Lopes ACS, Caiaffa WT, Sichieri R, MAS, LimaCosta MF. Consumo de nutrientes em adultos e idosos em estudo de base populacional: projeto bambuí. Cad Saude Publica 2005; 21(4):1201-1209.

22. Ferreira VA, Magalhães R. Práticas alimentares cotidianas de mulheres obesas moradoras da Favela da Rocinha (Rio de Janeiro, RJ, Brasil). Cien Saude Colet 2011; 16(6):2983-2991.

23. Schlindwein MM, Kassouf AL. Análise da influência de alguns fatores socioeconômicos e demográficos no consumo domiciliar de carnes no Brasil. RER 2006; 44(3):549-572.

24. Sato APS, Fujimori E, Szarfarc SC, Borges ALV, Tsunechiro MA. Consumo alimentar e ingestão de ferro de gestantes e mulheres em idade reprodutiva. Rev Latino-Am Enfermagem 2010; 18(2):247-254.

25. Brasil. Ministério da Saúde (MS). Carência de micronutrientes. Brasília: MS; 2007. (Cadernos de atenção Básica, n²0).

26. Suliburska J. A comparison of levels of select minerals in scalp hair samples with estimated dietary intakes of these minerals in women of reproductive age. Biological Trace Element Research 2011; 144(13):77-85.

27. Montilla RNG, Marucc MFN, Aldrighi JM. Avaliação do Estado Nutricional e do Consumo Alimentar de Mulheres no Climatério. Rev Assoc Med Bras 2003; 49(1):91-95.

28. Ilow R, Regulska-Ilow B, Ró ¿añska D, Zatoñska K, Dehghan M, Zhang X, Szuba A, Vatten L, JanikKoncewicz K, Mañczuk M, Zatoñski WA. Evaluation of mineral and vitamin intake in the diet of a sample of Polish population: baseline assessment from the prospective cohort 'PONS' study. Annals of Agricultural and Environmental Medicine 2011; 18(2):235-240.

Artigo apresentado em 16/07/2013

Aprovado em 27/08/2013

Versão final apresentada em 08/09/2013 